\title{
Adaptive tight frame based multiplicative noise removal
}

\author{
Weifeng Zhou ${ }^{1,2^{*}}$, Shuguo Yang ${ }^{1}$, Caiming Zhang ${ }^{2}$ and Shujun $\mathrm{Fu}^{3}$
}

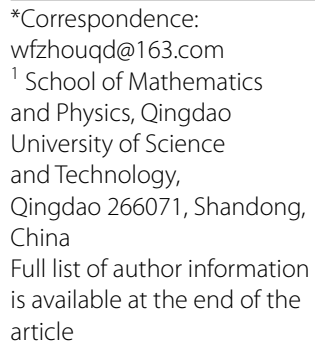

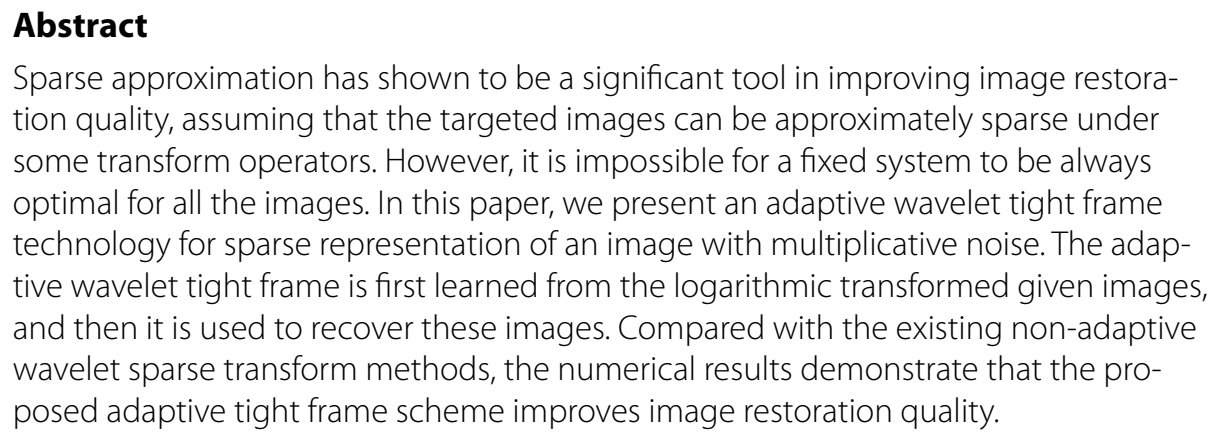

Sparse approximation has shown to be a significant tool in improving image restoration quality, assuming that the targeted images can be approximately sparse under some transform operators. However, it is impossible for a fixed system to be always optimal for all the images. In this paper, we present an adaptive wavelet tight frame technology for sparse representation of an image with multiplicative noise. The adaptive wavelet tight frame is first learned from the logarithmic transformed given images, and then it is used to recover these images. Compared with the existing non-adaptive wavelet sparse transform methods, the numerical results demonstrate that the proposed adaptive tight frame scheme improves image restoration quality.

\section{Background}

Noise is usually inevitable during data acquisition, transmission and record with CCD sensors etc. Image denoising as the most fundamental problem in image processing field is used to enhance images by reducing any degradations. Generally speaking, noise can be classified into additive noise and multiplicative noise based on the relationship of the noise and the signal. Unlike additive noise, multiplicative noise (e.g. speckle random noise) intensity is in proportion to the absolute image intensity and it mainly appears in synthetic aperture radar (SAR), laser imaging, ultrasound imaging, and positron emission tomography (PET) etc. (Goodman 2007; Oliver and Quegan 1998; Wagner et al. 1983) and has been paid great attention in recent years (e.g. Shi and Osher 2008; Aubert and Aujol 2008; Durand et al. 2010; Huang et al. 2012; Yu and Acton 2002; Steidl and Teuber 2010; Bioucas-Dias and Figueiredo 2010; Teuber and Lang 2012; Krissian et al. 2007). Mathematically, the degraded image $f$ corrupted by multiplicative noise $\delta$ usually can be formulated as

$$
f=u \cdot \delta
$$

Techniques and algorithms have been developed for solving this inverse problem to obtain an approximate image $u^{*}$ to the original image $u$ in recent years. Thereinto sparsity regularization methods are effective methods of them and have been widely used in every branch of image processing based on the hypothesis that images are approximately sparse under some transform domain $W$. Various linear operators that used to sparsely represent images are designed. For example, $W$ can be chosen as the first-order discrete

(c) 2016 Zhou et al. This article is distributed under the terms of the Creative Commons Attribution 4.0 International License (http:// creativecommons.org/licenses/by/4.0/, which permits unrestricted use, distribution, and reproduction in any medium, provided you give appropriate credit to the original author(s) and the source, provide a link to the Creative Commons license, and indicate if changes were made. 
gradient operator (Rudin et al. 1992). However, these given linear operators are not optimal for all the images. Then some dictionary leaning methods that were adapted to the reference images were proposed to improve the image restoration quality, e.g., the socalled K-SVD method (Donoho and Elad 2003; Elad and Ahron 2006; Mairal et al. 2007) and the data-driven tight frame method (Cai et al. 2014). The data-driven tight frame method outperforms the K-SVD method in terms of computational efficiency. These adaptive methods could usually provide better sparse approximations by deriving adaptive discrete dictionaries or framelets from the input reference images.

The multiplicative problem can be converted into an additive one by taking the log of both sides of (1) (Shi and Osher 2008), i.e., the following so-called Log-TV model

$$
\log f=\log u+\log \delta .
$$

Then the multiplicative noise removal scheme can be modeled by penalizing the sparse transform of $\log u$ instead of $u$ based on the noisy observation $\log f$. Motivated by the data-driven tight frame scheme for additive noise removal (Cai et al. 2014), we propose the adaptive tight frame approach for multiplicative noise removal in this paper. We first construct the adaptive wavelet tight frame based on the logarithmic transformed given image and then recover the image of interest by solving a wavelet balanced approach using the constructed adaptive tight frame system. We perform this adaptive regularization method for images contaminated by speckle noise and the simulation results suggest that the proposed adaptive tight frame method improves the image restoration quality especially reducing the artifacts compared with the traditional non-adaptive one.

The reminder of this work is arranged as follows: the definitions and constructions of the wavelet tight frame are provided in section "Construction of the wavelet tight frame". Then the scheme of learning the adaptive tight frame is given in the following section "Adaptive framelet". We present the adaptive framelet algorithm for multiplicative noise removal in section "Multiplicative noise removal method based on adaptive tight framelet". In Section "Numerical results", the representative simulation results are reported. Finally, this work briefly concludes in section "Conclusion".

\section{Construction of the wavelet tight frame}

\section{D discrete wavelet tight frame}

In this section, we first introduce the construction of the one-dimensional wavelet tight frame, i.e., its decompose and reconstruction. The corresponding 2D framelet is obtained by the tensor product of 1D framelets. More details can be seen in (Dong and Shen 2010; Chan and Shen 2003; Chan et al. 2004; Shen 2010).

A redundant tight frame in $\mathbb{R}^{N}$ is a sequence $\left\{x_{i}\right\}_{i=1}^{M}$ that satisfies

$$
\sum_{i=1}^{M}\left|\left\langle x_{i}, g\right\rangle\right|^{2}=\|g\|_{2}^{2}, \quad \forall g \in \mathbb{R}^{N} .
$$

Actually, redundant tight frame $(M \geq N)$ defined above is a generalization of orthogonal basis in $\mathbb{R}^{N}$, where $M=N$ and $\left\{x_{i}\right\}_{i=1}^{N}$ are linearly independent. There are two operators associated to the wavelet tight frame, i.e., analysis operator and synthesis operator. The analysis operator $W$ is written as 


$$
W=\left[x_{1}, x_{2}, \ldots, x_{M}\right]^{T} .
$$

For a signal $g, W g=\left\{\left\langle g, x_{i}\right\rangle\right\}_{i=1}^{M}$ denote the wavelet coefficients which are the inner products of $x_{i}$ and $g$. The synthesis operator denoted by $W^{T}$ is used to synthetize the the wavelet coefficients $s$ by $W^{T} s=\sum_{i=1}^{M} s(i) x_{i}$. Then the identity (3) is equivalent to

$$
W^{T} W=I_{N},
$$

where $I_{N}: \mathbb{R}^{N} \rightarrow \mathbb{R}^{N}$ denotes the identity operator.

Multi-resolution analysis (MRA) can be used to construct wavelet tight frame that associated with a so-called refinement mask $h_{0}$ and a class of MRA-based wavelet tight framelets are constructed (Dong and Shen 2010; Chan and Shen 2003; Chan et al. 2004; Shen 2010). For a signal $g \in \mathbb{R}^{N}$, the discrete wavelet operator $W$ associated with filters $\boldsymbol{h}=\left\{h_{0}, h_{1}, \ldots, h_{r-1}\right\}$ is defined as follows

$$
W(\boldsymbol{h}): g \in \mathbb{R}^{N} \rightarrow\left[\begin{array}{c}
h_{0} * g \\
\vdots \\
h_{r-1} * g
\end{array}\right] \in \mathbb{R}^{r N},
$$

where $*$ denotes the filtering procedure. $h_{0}$ denotes the low-pass filter which satisfies $\sum_{j} h_{0}(j)=1$, and $h_{i}, i=1, \ldots, r-1$ satisfying $\sum_{j} h_{i}(j)=0$ is called high-pass filters usually. The multi-level wavelet operator can be obtained by using this one-level wavelet $W(\boldsymbol{h})$ recursively to the low-pass coefficients. More details can be seen in (Chan and Shen 2003; Chan et al. 2004). The so-called perfect reconstruction property, i.e., $W(\boldsymbol{h})^{T} W(\boldsymbol{h})=I_{N}$ is equivalent to the following Unitary Extension Principle condition (Han et al. 2011):

$$
\sum_{i=0}^{r-1} \sum_{l \in \mathbb{Z}} h_{i}(m+l) h_{i}(l)=\delta_{m}, \quad \forall m \in \mathbb{Z} .
$$

Here $\delta_{m}=1$ if $m=0$ and $\delta_{m}=0$ otherwise.

\section{Adaptive framelet}

Choosing a fixed redundant system that performs well for all the images is rather difficulty, the dictionary learning approaches that are adapted to the images have been explored recently (Donoho and Elad 2003; Elad and Ahron 2006; Mairal et al. 2007; Cai et al. 2014). The well-known K-SVD method (Elad and Ahron 2006; Mairal et al. 2007) is such a representative work with the advantage of better approximating images with abundant textures compared with the non-learning schemes. Although K-SVD method outperforms the traditional non-learning ones, the poor computational efficiency results in the difficulty in practical application. Recently, Cai et al. (2014) proposed the datadriven tight frame construction scheme. On one hand, this designed adaptive tight frame scheme reduces the artifacts which usually exists in the images processed by the fixed wavelet tight frames, on the other hand, the minimization problem of learning the adaptive wavelet tight frame is high-efficiency compared with the K-SVD learning scheme. This adaptive framelet method has been used in CT image reconstruction (Zhou et al. 
2013) and seismic data processing (Liang et al. 2014) etc. Next, we will first present the method of constructing the adaptive wavelet tight frame.

Let $\boldsymbol{H}=\left\{H_{i}\right\}_{i=0}^{R-1}$ denote a family of two-dimensional filters. Then the 2D wavelet transform operator can be defined as

$$
W(\boldsymbol{H}): g \in \mathbb{R}^{N^{2}} \rightarrow\left[\begin{array}{c}
H_{0} * g \\
\vdots \\
H_{R-1} * g
\end{array}\right] \in \mathbb{R}^{R N^{2}} .
$$

Here, we use $*$ to denote the filtering of two $2 \mathrm{D}$ arrays.

In Cai et al. (2014), the data-driven tight frame construction scheme was introduced in order to obtain better sparse representation of the given signal $g$ by solving

$$
\min _{s, \boldsymbol{H}}\left\|s-W_{a}(\boldsymbol{H}) g\right\|_{2}^{2}+\mu^{2}\|s\|_{0}, \quad \text { s.t. } \quad W_{a}(\boldsymbol{H})^{T} W_{a}(\boldsymbol{H})=I_{N^{2}},
$$

where $W_{a}$ denotes the adaptive wavelet tight frame that satisfies $W_{a}^{T} W_{a}=I$. The first term in (6) is to ensure that the coefficients $s$ are close to $W_{a} g$, and the second term is to make the coefficients $s$ be sparse. Then adaptive 2D filters $\boldsymbol{H}=\left\{H_{i}\right\}_{i=0}^{R-1}$ and the approximated sparse coefficients $s$ can be obtained by solving (6). Based on the alternating minimization principle, (6) can be solved by solving two sub-problems, i.e.,

$$
\begin{aligned}
& \min _{s}\left\|s-W_{a}(\boldsymbol{H}) g\right\|_{2}^{2}+\mu^{2}\|s\|_{0}, \\
& \min _{\boldsymbol{H}}\left\|s-W_{a}(\boldsymbol{H}) g\right\|_{2}^{2}, \text { s.t. } W_{a}(\boldsymbol{H})^{T} W_{a}(\boldsymbol{H})=I_{N^{2}} .
\end{aligned}
$$

Observing that (7) is used to learn the adaptive tight frame based on the given image g. Obviously, the analytical solution of the $s$-subproblem (7) can be obtained via hard thresholding. More details about hard thresholding can be seen in Blumensath and Davies $(2009,2010)$. The second sub-problem (8) is a complex non-convex minimization problem with the quadratic constraints that can be simplified as

$$
\sum_{i=0}^{R-1} \sum_{n \in \mathbb{Z}^{2}} H_{i}(k+n) H_{i}(n)=\delta_{k}, \quad \forall k \in \mathbb{Z}^{2},
$$

where $\delta_{k}=1$ if $k=(0,0)$ and $\delta_{k}=0$ otherwise Han et al. (2011). Then the wavelet tight frame can be obtained by solving the following minimization problem with orthogonal constraints:

$$
\min _{\boldsymbol{H}}\left\|s-W_{a}(\boldsymbol{H}) g\right\|_{2}^{2} \text {, s.t. }\left\langle H_{i}, H_{j}\right\rangle=\frac{1}{r^{2}} \delta_{i-j}, \quad 0 \leq i, j \leq R-1 .
$$

Here $R=r \times r$ is also the support of $H_{i}, i=0, \ldots, R-1$. This problem (10) can be solved exactly via the singular value decomposition (SVD) (Cai et al. 2014; Zou et al. 2006). Concretely, partitioning the coefficient vector $s$ into $r^{2}$ vectors, denoted by 
$s_{i} \in \mathbb{R}^{N^{2} \times 1}, i=1,2, \ldots r^{2}$, corresponding to the coefficient obtained from the filter $H_{i}$. Define

$$
\begin{aligned}
\tilde{S} & =\left[\begin{array}{ccc}
s_{0}(1) & \ldots & s_{r^{2}-1}(1) \\
\vdots & \vdots & \vdots \\
s_{0}\left(N^{2}\right) & \ldots & s_{r^{2}-1}\left(N^{2}\right)
\end{array}\right] \in \mathbb{R}^{N^{2} \times r^{2}} \\
\tilde{G} & =\left[g_{1}, g_{2}, \cdots g_{N^{2}}\right] \in \mathbb{R}^{r^{2} \times N^{2}} \\
\tilde{H} & =\left[H_{0}, H_{1}, \ldots, H_{r^{2}-1}\right] \in \mathbb{R}^{r^{2} \times r^{2}}
\end{aligned}
$$

where $g_{i}, i=0, \ldots, N^{2}-1$, denotes the partition of $g$ corresponding to the filter size $r$. Then the minimization (10) can be solved by solving the following problem

$$
\max _{\tilde{H}} \operatorname{Tr}\left(\tilde{H} \tilde{S}^{T} \tilde{G}^{T}\right), \text { s.t. } \tilde{H}^{T} \tilde{H}=\frac{1}{r^{2}} I_{r^{2}} .
$$

Theorem 1 Zou et al. (2006) Let $C_{m \times r}$ denotes matrix with rank $r$ and the SVD decomposition of matrix $A_{m \times r}$ is $A=U D Q^{T}$, Then $C_{*}=U Q^{T}$ is the solution of the following constrained maximization problem:

$$
\max _{C} \operatorname{Tr}\left(C^{T} A\right) \text {, s.t. } C^{T} C=I_{r^{2}} .
$$

Let $A=\tilde{S}^{T} \tilde{G}^{T}=U D Q^{T}$. Then by Theorem 1 , the $W_{a^{-}}$subproblem can be obtained by the following equation

$$
\left[H_{0}, H_{1}, \ldots, H_{r^{2}-1}\right]=\frac{1}{r} Q U^{T} .
$$

\section{Multiplicative noise removal method based on adaptive tight framelet}

The wavelet based sparse representation methods for Gaussian noise removal can be usually summarized as

$$
s^{*}=\arg \min _{s} \frac{1}{2}\left\|W^{T} s-f\right\|_{2}^{2}+\frac{\tau}{2}\left\|\left(I-W W^{T}\right) s\right\|_{2}^{2}+\lambda\|s\|_{1} .
$$

Then the recovered image $u^{*}$ approximates $W^{T} s^{*}$. Here, the second term $\frac{\tau}{2}\left\|\left(I-W W^{T}\right) s\right\|_{2}^{2}$ is used to balance the distance between the coefficient $s$ and the corresponding recovered signal $W^{T}$, so three categories are distinguished by different values of $\tau$. When $\tau=0$, the minimization (15) is called the synthesis based approach (Cai et al. 2009; Starck et al. 2005). Many effective iterative algorithms have sprung up to overcome the computational difficulty caused by the non-differentiable regularization term, e.g., split Bregman method (Goldstein and Osher 2009; Cai et al. 2010), alternating direction method (Afonso et al. 2010), forward-backward algorithm (Combettes and Pesquet 2007), primal-dual algorithm (Chan et al. 1999), etc. When $\tau=\infty$, the model (15) is just the analysis based approach (Daubechies et al. 2004). When $0<\tau<\infty$, the above model is called a balanced approach (Cai et al. 2009; Chan et al. 2003). The three methods are equivalent if the transform operator $W$ is orthogonal.

In this work, we will take the balanced approach for avoiding the multiple iterations produced in solving analysis based approach so as to reduce the computing consuming. 
The balanced approach yields the restoration result that balanced the sparsity of the associated tight framelet coefficients and the regularity of the recovered result. The degenerated image can be recovered by the following formula based on the wavelet tight framelet $W(\boldsymbol{H})=W\left(H_{0}, H_{1}, \ldots, H_{r^{2}-1}\right)$ balanced approach

$$
z=W^{T}\left(T_{\lambda}(W(\log f))\right), u=\exp (z) .
$$

That is the following algorithm

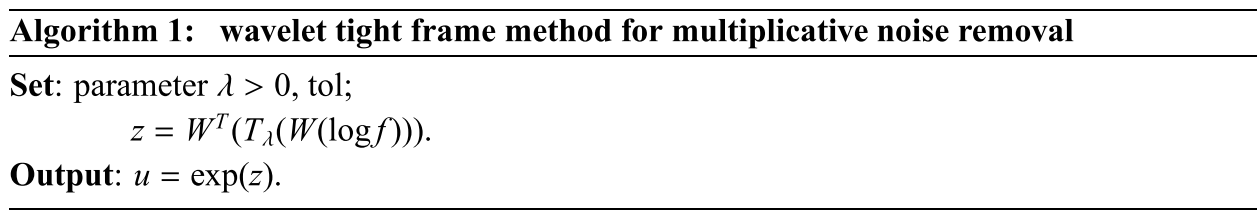

The constructed adaptive framelets were used in Gaussian noise removal and demonstrated their superiority in terms of image restoration quality compared with the corresponding non-adaptive ones (Cai et al. 2014) and improved computational efficiency compared with the K-SVD learning method. In this section, we will generalize the adaptive framelet method to multiplicative noise removal by considering the multiplicative denoising problem in log-domain. We convert multiplicative denoising problem into additive noise based one in log-domain. Then the logarithmic adaptive tight framelet constructed based on the noisy image in the $\log$-domain, i.e., $\log f$ instead of $f$, is used for sparse representation so as to improve the recovered images quality. We summarize the adaptive tight framlet balanced approach for denoising the degenerated images contaminated by multiplicative noise as the following Algorithm 2.

\section{Algorithm 2: Adaptive wavelet tight frame algorithm for multiplicative noise removal}

Set: parameters $\tilde{\lambda}>0, \mu>0$, tol;

Initialization: 2D initial tight frame filters $\boldsymbol{H}^{(0)}=\left\{H_{i}^{(0)}\right\}_{i=0}^{r^{2}-1}$.

(I) Generate 2D adaptive wavelet tight frame $W_{a}$ from a log observed image $\log f$

For $l=0,1,2, \cdots, L-1$

(a) construct $W_{a}\left(\boldsymbol{H}^{(l)}\right)$ with filters $\boldsymbol{H}^{(\ell)}=\left\{H_{i}^{l}\right\}_{i=0}^{r^{2}-1}$;

(b) let $s^{l}=T_{\mu}\left(W_{a}\left(\boldsymbol{H}^{(L)}\right) g\right)$, where $T_{\mu}$ denote the tranditional thresholding operator;

(c) define matrix $\tilde{S}, \tilde{G}$ by (11);

(d) SVD for $\tilde{S}^{T} \tilde{G}^{T}$, s.t. $\tilde{S}^{T} \tilde{G}^{T}=U D Q^{T}$;

(e) $\boldsymbol{H}^{(l+1)}=\left\{H_{i}^{(l+1)}\right\}_{i=0}^{2^{2}-1}$, where $\left[H_{0}^{(l+1)}, H_{1}^{(l+1)}, \ldots, H_{r^{2}-1}^{(l+1)}\right]=\frac{1}{r} Q U^{T}$.

End

(П) denoising based on the above learned adaptive operator

Adaptive Tight Frame based Multiplicative Noise Removal

construct $W_{a}=W_{a}\left(\boldsymbol{H}^{(L)}\right)$ with filters $\boldsymbol{H}^{(L)}=\left\{H_{i}^{(L)}\right\}_{i=0}^{r^{2}-1}$; $z=W_{a}^{T}\left(T_{\bar{\lambda}}\left(W_{a}(\log f)\right)\right)$.

Output: $u=\exp (z)$. 


\section{Numerical results}

This section will illustrate the restoration results by wavelet based multiplicative noise removal approaches. Furthermore, the superiority of the proposed adaptive wavelet based scheme (Algorithm 2) will be illustrated here through simulations on frequentlyused test images "lenna" and "barbara". The following peak signal to noise ratio (PSNR) is used to evaluate the recovery quality

$$
\operatorname{PSNR}=20 \log 10 \frac{255}{\frac{1}{M N}\left\|x-x_{0}\right\|_{2}},
$$

where $x$ and $x_{0}$ respectively denotes the recovered image and the original image with dimension $M \times N$. Larger PSNR value usually means better image restoration quality. We perform all the simulations on the PC with $2.9 \mathrm{HZ}$ and 64-bit operator system in the circumstance of 2009 MATLAB.

Example 5.1 Figure 1 shows the recovered results by non-adaptive wavelet tight framelet and adaptive wavelet tight framelet approach based on the ground truth image "lenna" (Fig. 1a). "lenna" was polluted by multiplicative speckle noise with noise variance $\sigma=0.1$ (see Fig. 1b). Note that we can directly use the noisy image, i.e., Fig. 1b, as the reference image to learn the adaptive wavelet tight framelet operator. Figure 1c, $\mathrm{d}$ respectively illustrate the reconstructed images by non-adaptive wavelet framelet and adaptive wavelet framelet with the $8 \times 8$ Haar wavelet as the initial filter. The difference images are respectively illustrated in Fig. 1e,f. It can be seen that adaptive framelet approach (Fig. 1d) can suppress artifacts and capture more details than the corresponding non-adaptive one (Fig. 1c), which also can be seen in difference images via comparing Fig. If by adaptive framelet approach with Fig. 1e by non-adaptive method. The PSNR values listed in Table 1 also reflect that adaptive framelet approach yields better recovered results than the corresponding non-adaptive one. In Table 1, we also compare the results by adaptive tight framelet approaches in terms of different initial filter sizes and filter types with the results by corresponding non-adaptive ones. Data in Table $1 \mathrm{dem}$ onstrates that adaptive tight framelet approaches defeat the corresponding non-adaptive ones with respect to image restoration quality evaluated by PSNR. Generally speaking, larger filter size means better restoration quality in terms of the tested filter size. The computation time for recovering a $256 \times 256$ image by a $3 \times 3,7 \times 7$, and $15 \times 15$ adaptive tight frame is respectively about $0.16,1.48$ and $14.75 \mathrm{~s}$, which is more excellent than the K-SVD in terms of efficiency. Here and in the following test, the parameters used for learning the adaptive wavelet framelet and thresholding denoising follow the selection rule provided in Cai et al. (2014), that is $\alpha=5.1 \sigma$ and $\widetilde{\lambda}=2.6 \sigma$.

Example 5.2 "barbara" (Fig. 2a) is usually used to assess the ability of catching textures for different algorithms owing to its abundant textures. Figure $2 \mathrm{~b}$ is the degenerated "barbara" with multiplicative speckle noise variance $\sigma=0.1$. Figure 2c, d respectively present the recovered images by non-adaptive wavelet framelet and adaptive wavelet framelet with the $8 \times 8$ Haar wavelet as the initial filter. Figure $2 \mathrm{e}, \mathrm{f}$ are the corresponding difference images respectively. Obviously, adaptive framelet approach (Fig. 3d) has the advantages in preserving textures and suppressing artifacts compared with the 


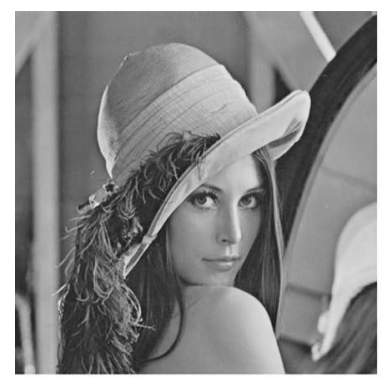

a original

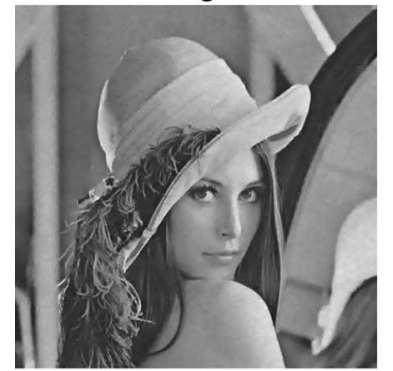

c non-adaptive wavelet

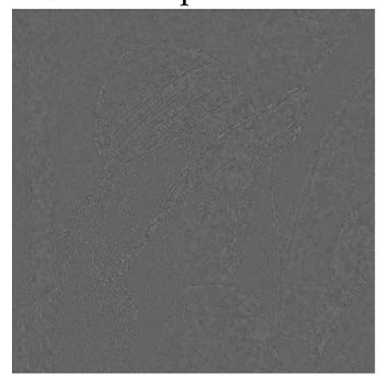

e difference by non-adaptive wavelet

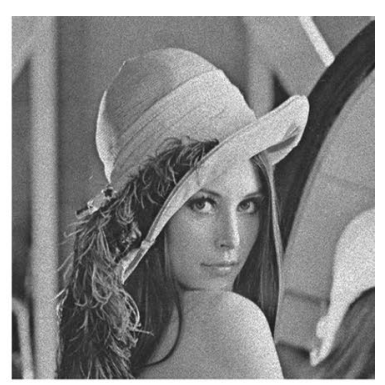

b noise image

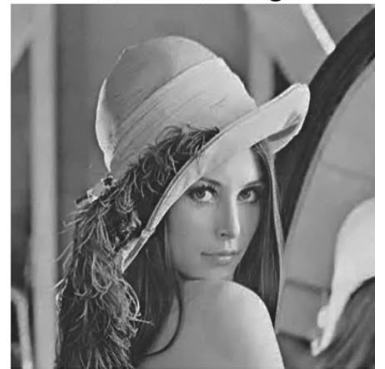

d adaptive wavelet

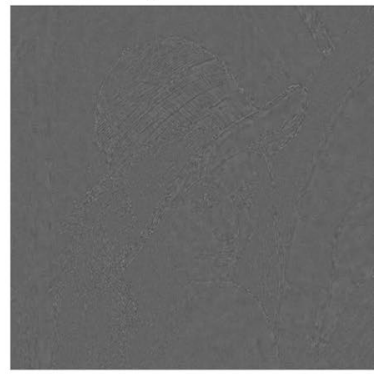

f difference by adaptive wavelet

Fig. 1 Denoised results for polluted "lenna" by adaptive wavelet tight frame and non-adaptive wavelet tight frame. a Denotes the original image and $\mathbf{b}$ is the noisy image. $\mathbf{c}$, $\mathbf{d}$ are respectively the recovered images by adaptive wavelet tight frame method and the corresponding non-adaptive one with $8 \times 8$ Haar wavelet. $\mathbf{e}=$ $\mathbf{a}-\mathbf{c}$ and $\mathbf{f}=\mathbf{a}-\mathbf{d}$ are the corresponding difference images

Table 1 Denoised results for polluted "lenna" by adaptive wavelet tight frames and the corresponding non-adaptive ones in terms of different filter sizes

\begin{tabular}{llll}
\hline Initial tight frame & Filter size & Non-adaptive algorithm & Adaptive algorithm \\
\hline \multirow{2}{*}{ Haar wavelet } & $2 \times 2$ & 31.25 & 31.54 \\
& $4 \times 4$ & 32.91 & 33.89 \\
Local DCT & $8 \times 8$ & 33.16 & 34.64 \\
& $2 \times 2$ & 31.26 & 31.45 \\
Linear framelet & $4 \times 4$ & 33.57 & 33.85 \\
& $8 \times 8$ & 34.32 & 34.68 \\
& $3 \times 3$ & 32.07 & 33.26 \\
& $7 \times 7$ & 33.12 & 34.63 \\
\hline
\end{tabular}




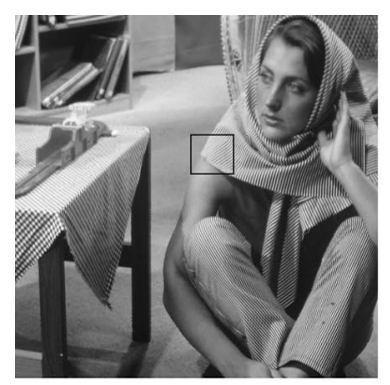

a original

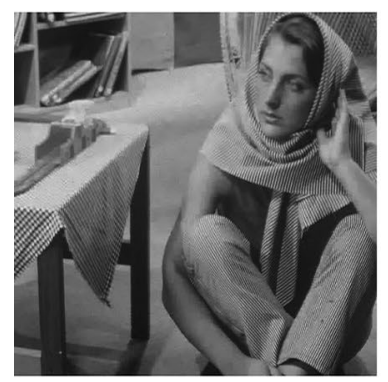

c non-adaptive wavelet

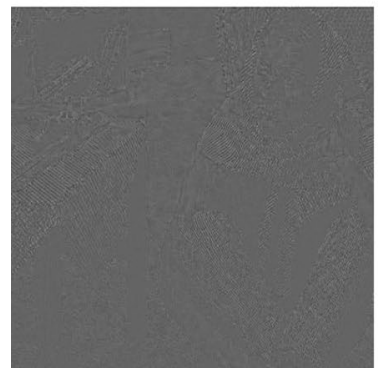

e difference by non-adaptive wavelet

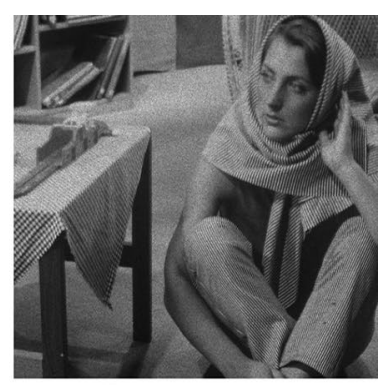

b noise image

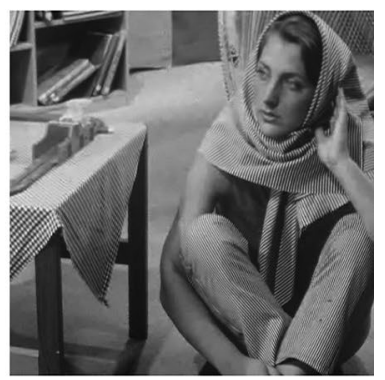

d adaptive wavelet

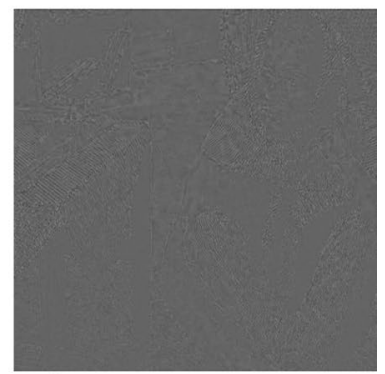

f difference by adaptive wavelet

Fig. 2 Denoised results for polluted "barbara" by adaptive wavelet tight frame and non-adaptive wavelet tight frame. $\mathbf{a}$ is the original image and $\mathbf{b}$ is the noisy image. $\mathbf{c}, \mathbf{d}$ are respectively the recovered images by adaptive wavelet tight frame method and the corresponding non-adaptive one with $8 \times 8$ Haar wavelet. $\mathbf{e}=$ $\mathbf{a}-\mathbf{c}$ and $\mathbf{f}=\mathbf{a}-\mathbf{d}$ are respectively the difference image

non-adaptive scheme (Fig. 3c) from the zoomed part of "barbara" (Fig. 3). Repeatedly, we compare the results by adaptive tight framelet approaches in terms of different initial filter sizes and filter types with the results by corresponding non-adaptive ones (see Table 2).

Table 3 lists the SNR results of the adaptive B-Spline wavelet framelet method in terms of larger speckle noise with variance $\sigma=0.2$. From Table 3, we can see that our algorithm can also obtain better results than the corresponding non-adaptive ones.

\section{Conclusion}

In this paper, we have generalized the adaptive tight frame methods to multiplicative noise removal problem. We learn the image-specific wavelet tight framelet based on the logarithmic transformed image by using SVD based explicit formulas. Numerical 


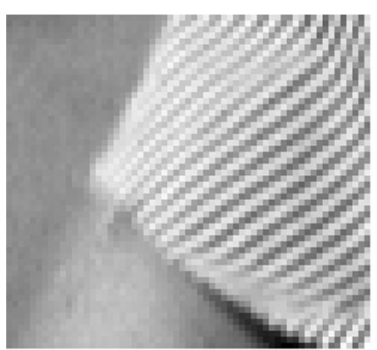

a original

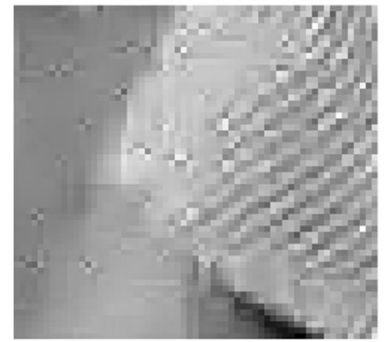

c non-adaptive wavelet

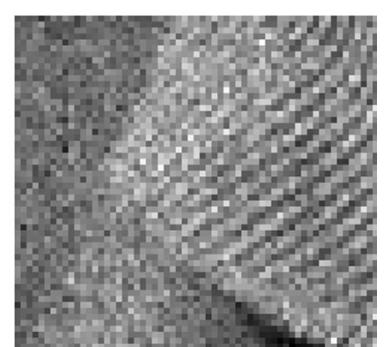

b noise image

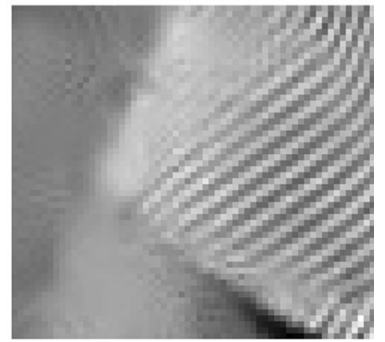

d adaptive wavelet

Fig. 3 The zoomed denoised results for "barbara", a is the original zoomed image and $\mathbf{b}$ is the zoomed noisy image. $\mathbf{c}, \mathbf{d}$ are respectively the zoomed recovered images by adaptive wavelet tight frame method and the corresponding non-adaptive one

Table 2 Denoised results for polluted "barbara" by wavelet tight frame method and adaptive wavelet tight frame in terms of different filter sizes

\begin{tabular}{llll}
\hline Initial tight frame & Filter size & Non-adaptive algorithm & Adaptive algorithm \\
\hline Haar wavelet & $2 \times 2$ & 29.44 & 29.55 \\
& $4 \times 4$ & 30.18 & 31.39 \\
Local DCT & $8 \times 8$ & 30.46 & 32.72 \\
& $2 \times 2$ & 29.37 & 29.48 \\
Linear framelet & $4 \times 4$ & 31.27 & 31.55 \\
& $8 \times 8$ & 32.32 & 32.71 \\
& $3 \times 3$ & 29.91 & 30.94 \\
& $7 \times 7$ & 30.63 & 32.41 \\
\hline
\end{tabular}

Table 3 Larger noise removal results by our proposed adaptive wavelet tight frame and the corresponding non-adaptive one

\begin{tabular}{llll}
\hline Image & Filter size & Non-adaptive algorithm & Adaptive algorithm \\
\hline \multirow{3}{*}{ Lenna } & $3 \times 3$ & 26.64 & 27.58 \\
& $7 \times 7$ & 26.96 & 28.96 \\
Barbara & $3 \times 3$ & 24.75 & 25.81 \\
\hline
\end{tabular}


experiments demonstrate that the derived adaptive tight frame balanced based regularization method improves the image restoration quality compared with the corresponding non-adaptive one not only in enhancing PSNR value but also in preserving fine image features.

\title{
Authors' contributions
}

WZ drafted the manuscript. SY, CZ, and SF made some revisions of the manuscript. All authors read and approved the final manuscript.

\author{
Author details \\ 1 School of Mathematics and Physics, Qingdao University of Science and Technology, Qingdao 266071, Shandong, China. \\ ${ }^{2}$ School of Computer Science and Technology, Shandong University, Jinan 250101, Shandong, China. ${ }^{3}$ School of Math- \\ ematics, Shandong University, Jinan 250100, China.
}

\section{Acknowledgements}

Weifeng Zhou was supported in part by NSFC (11526118), Natural Science Foundation of Shandong Province (ZR2015PF001), the Foundation of China Postdoctoral (2015M570594), the Department of Education Foundation of Yunnan Province (2014Y446) and the Talent Start-up Fund of Qingdao Science and Technology University (010022670); Shuguo Yang was supported by the Science and Technology Planning Project of Shandong Province (2015GGX101020); Shujun Fu was supported in part by NSFC (61272239), the Science and Technology Development Project of Shandong Province of China (2014GGX101024), and the foundamentational Research Funds of Shandong University (2014JC012).

\section{Competing interests}

The authors declare that they have no competing interests.

Received: 18 September 2015 Accepted: 21 December 2015

Published online: 12 February 2016

\section{References}

Afonso M, Biouscas-Dias J, Figueiredo M (2010) Fast image recovery using variable splitting and constrained optimization. IEEE Trans Image Process 19:2345-2356

Aubert G, Aujol J-F (2008) A variational approach to remove multiplicative noise. SIAM J Appl Math 68(4):925-946

Bioucas-Dias JM, Figueiredo M (2010) Multiplicative noise removal using variable splitting and constrained optimization. IEEE Trans Image Process 19(7):1720-1730

Blumensath T, Davies ME (2009) Iterative hard thresholding for compressed sensing. Appl Comput Harmon Anal 27(3):265-274

Blumensath T, Davies ME (2010) Normalized iterative hard thresholding guaranteed stability and performance. IEEE J Sel Top Signal Process 4(2):298-309

Cai J-F, Ji H, Liu C et al (2009) Blind motion deblurring from a single image using sparse approximation. In: Proceedings of the IEEE computer society conference on computer vision and pattern recognition in 2009, pp 104-111. http://www.math.nus.edu.sg/ matzuows/blind_deblurring3.pdf

Cai J-F, Osher S, Shen Z (2010) Split Bregman methods and frame based image restoration. Multiscale Model Simul 8:337-369

Cai J-F, Huang S, Ji H, Shen Z, Ye G-B (2014) Data-driven tight frame construction and image. Appl Comput Harmon Anal 37(1):89-105

Chan TF, Golub GH, Mulet P (1999) A nonlinear primal-dual method for total variation-based image restoration. SIAM J Sci Comput 20:1964-1977

Chan RH, Chan TF, Shen L et al (2003) Wavelet algorithms for high-resolution image reconstruction. SIAM J Sci Comput 24(4):1408-1432

Chan RH, Riemenschneider SD, Shen L, Shen Z (2004) Tight frame: an efficient way for high-resolution image reconstruction. Appl Comput Harmon Anal 17:91-115

Chan $\mathrm{RH}$, Shen Z (2003) Wavelet algorithms for high-resolution image reconstruction. SIAM J Sci Comput 24:1408-1432

Combettes PL, Pesquet J-C (2007) A Douglas-Rachford splitting approach to nonsmooth convex variational signal recovery. IEEE J Sel Top Signal Process 1:564-574

Daubechies I, Defrise M, De Mol C (2004) An iterative thresholding algorithm for linear inverse problems with a sparsity constraint. Commun Pure Appl Math 57(11):1413-1457

Dong B, Shen Z (2010) MRA Based Wavelet Frames and Applications, IAS Lecture Notes Series, Summer Program on "The Mathematics of Image Processing" Park City Mathematics Institute

Donoho DL, Elad M (2003) Maximal sparsity representation via $I_{1}$ minimization. Proc Natl Acad Sci 100:2197-2202

Durand S, Fadili J, Nikolova M (2010) Multiplicative noise removal using L1 fidelity on frame coefficients. J Math Imaging Vis 36(3):201-226

Elad M, Ahron M (2006) Image denoising via sparse and redundant representations over learned distionaries. IEEE Trans Image Process 54:3736-3745

Goldstein T, Osher S (2009) The split Bregman algorithm for L1 regularized problems. SIAM J Imaging Sci 2:323-343

Goodman J (2007) Speckle phenomena in optics: theory and applications. Roberts and Company Publication, Greenwood Village 
Han B, Kutyniok G, Shen Z (2011) Adaptive multiresolution analysis structures and shearlet systems. SIAM J Numer Anal 49:1921-1946

Huang Y, Moisan L, Ng MK et al (2012) Multiplicative noise removal via a learned dictionary. IEEE Trans Image Process 21(11):4534-4543

Krissian K, Westin CF, Kikinis R et al (2007) Oriented speckle reducing anisotropic diffusion. IEEE Trans Image Process 16(5):1412-1424

Liang J, Ma J, Zhang X (2014) Seismic data restoration via data-driven tight frame. Geophysics 79(3):65-74

Mairal J, Elad M, Sapiro G (2007) Sparse representation for color image restoration. IEEE Trans Image Process 17:53-69

Oliver C, Quegan S (1998) Understanding synthetic aperture radar images. Artech House, Norwood

Rudin L, Osher S, Fatemi E (1992) Nonlinear total variation based noise removal algorithms. Phys D 60:259-268

Shen Z (2010) Wavelet frames and image restorations. In: Proceedings of the international congress of mathematicians in 2010. Hyderabad, India, pp 2834-2863

Shi J, Osher S (2008) A nonlinear inverse scale space method for a convex multiplicative noise model. SIAM J Imaging Sci 1(3):294-321

Starck JL, Elad M, Donoho DL (2005) Image decomposition via the combination of sparse representations and a variational approach. IEEE Trans Image Process 14(10):1570-1582

Steidl G, Teuber T (2010) Removing multiplicative noise by Douglas-Rachford splitting methods. J Math Imaging Vis 36(2):168-184

Teuber T, Lang A (2012) Nonlocal filters for removing multiplicative noise. Scale Space and Variational Methods in Computer Vision. Springer, Berline, p 50-61

Wagner RF, Smith SW, Sandrik JM (1983) Statistics of speckle in ultrasound B-scans. IEEE Trans Sonics Ultrason 30(3):156-163

Yu Y, Acton ST (2002) Speckle reducing anisotropic diffusion. IEEE Trans Image Process 11(11):1260-1270

Zhou W, Cai J-F, Gao H (2013) Adaptive tight frame based medical image reconstruction: a proof-of-concept study for computed tomography. Inverse Probl 29:125006

Zou H, Hastie T, Tibshirani R (2006) Sparse principle component analysis. J Comput Graph Stat 15:265-86

\section{Submit your manuscript to a SpringerOpen ${ }^{\circ}$ journal and benefit from:}

- Convenient online submission

- Rigorous peer review

- Immediate publication on acceptance

- Open access: articles freely available online

- High visibility within the field

- Retaining the copyright to your article

Submit your next manuscript at $>$ springeropen.com 